\title{
BEVOLKERUNGSPROBLEME FRANKREICHS
}

\section{Von Fritz Nussbaum}

Frankreich weist Bevölkerungsprobleme auf, die schon seit langem das lebhafte Interesse vieler Kreise und die Besorgnis französischer Staatsmänner hervorriefen, und zahlreiche Autoren haben sich damit beschäftigr und auseinandergesetz ${ }^{1}$. Als besonders beunruhigende Tatsache ist der anhaltende Geburtenrückgang der französischen Nation und die Abnahme der Bevölkerung zahlreicher Departemente in Erscheinung getreten. Dementsprechend ist die mittlere Volksdichte auffallend gering für ein Land, dessen Naturgrundlagen, wie man allgemein weiß, in jeder Hinsicht außerordentlich reich und günstig sind.

Sowohl von der Regierung wie von privaten Organisationen sind Schritte unternommen und Maßnahmen getroffen worden, um den volkswirtschaftlich unheilvollen Verhältnissen und Zuständen entgegenzutreten; leider sind diese Bestrebungen bis zur Stunde ohne nennenswerten Erfolg geblieben.

Die angedeuteten Bevölkerungsverhältnisse sollen im folgenden an Hand statistischer Erhebungen für einzelne Gebiete Frankreichs, speziell für die Departemente des Stammlandes, kurz erörtert werden, deren Zahl in der Zeit von 1860 bis 1919 zufolge geschichtlicher Vorgänge zwischen 83 und 88 geschwankt hat.

Die Volkszählung von 1821 ergab eine Gesamteinwohnerzahl von 30472000, die von 1841 eine solche von 34230000 und die von 1851 eine von 35781000. 1861 zählte man 37386000, nun mit Savoyen und Nizza. 1872 waren es aber 36100000 , nämlich ohne Elsaß und Lothringen. 1881 wurden 37773000 Einwohner gezählt. Demnach betrug die Bevölkerungszunahme für die Zeitspanne von 1821 bis 1841 $0,56 \%$, von 1841 bis $18610,25 \%$, von 1861 bis 1881 nur noch $0,19 \%$. Immer deutlicher machen sich von diesem Zeitpunkt an die Anzeichen eines anhaltenden Rückganges der französischen Nation bemerkbar.

Der Zuwachs der Bevölkerung oder der Geburtenüberschuß betrug im Jahre 1875 noch 160000 Menschen, im Jahre 1886 jedoch nur 52616; aber bereits 1890 zeigte sich ein Fehlbetrag von 33446 Menschen. Im Jahre 1896 betrug die Gesamtbevölkerung Frankreichs 38,5 Millionen Einwohner; bei einem Areal von $536400 \mathrm{~km}^{2}$ ergab sich eine mittlere Volksdichte von 71,8 per $\mathrm{km}^{2}$. Im Jahre 1911 war die Bewohnerzahl um 1,1 Million größer bei gleicher Arealausdehnung, nämlich 39,6 Millionen; aber diese Zunahme, die eine Volksdichte von 73,8 Einwohner per $\mathrm{km}^{2}$ bewirkte, war zur Hauptsache durch Zuwanderung bedingt. Im Jahre 1926 zählte Frankreich 40,4 Millionen Einwohner bei einem Areal von $550980 \mathrm{~km}^{2}$, letzteres also vermehrt um die seit dem ersten Weltkrieg wieder erworbenen Gebiete von Elsaß-Lothringen, deren Bevölkerung damals 1,16 Millionen Seelen betrug.

1931 hatte Frankreich 41834900, 194640517900 Einwohner. In den Jahren 1910 bis 1931 betrug die jährliche Bevölkerungszunahme Frankreichs nur 0,04\%, gegenüber $0,54 \%$ in Deutschland und $0,63 \%$ in Italien. Jener geringe Zuwachs ist jedoch bei der noch vorhandenen Auswanderung von Landeskindern nur durch Zuwanderung von Ausländern und nicht durch die natürliche Vermehrung der eigenen Nation bedingt.

Betrachten wir nun die Bevölkerungsverhältnisse der Departemente des französischen Stammlandes. Wie sich aus den Zusammenstellungen E. LevasSEURS der in Frankreich seit 1801 durchgeführten Volkszählungen ergibt ${ }^{2}$, haben die Be-

\footnotetext{
1 Man vergleiche u. a. die von E. Granger in "La France", Paris 1932, auf Seite 426 gegebene Autorenliste.

2 "Population et Démographie de la France." Grande Encyclopédie, vol. 14 et 17. Paris 1892.
} 
völkerungszahlen sozusagen sämtlicher Departemente bis gegen die Mitte des vorigen Jahrhunderts zugenommen, worauf bei einer großen Anzahl von Zählgebieten eine anhaltende Abnahme der Bewohner eingesetzt hat. Diese Abnahme beginnt bei einigen Departementen schon nach dem Jahre 1831, bei anderen nach 1841; bei 50 Departementen hat die Bevölkerung im Zähljahre 1851 das Maximum erreicht, um von da bis zur Gegenwart mehr oder weniger gleichmäßig abzunehmen. Ein weiteres, deutlich erkennbares Stichjahr scheint 1891 gewesen zu sein, in welchem noch 19 Departemente ihre maximale Einwohnerzahl aufgewiesen haben. Die nach der Volkszählung von 1931 berechnete mittlere Volksdichte beträgt für Frankreich bei einer Gesamteinwohnerzahl von 41,834 Millionen 76,1 Einwohner per $\mathrm{km}^{2}$. Allein 63 von 87 Departementen bleiben unter diesem Mittel. Es sind dies vor allem die Departemente des Zentralmassivs, der Alpen, mit Ausnahme der Alpes-Maritimes, ferner der Pyrenäen und die Landes; auch die Vogesen gehören zu den Departementen, die Volksdichtewerte von weniger als 50 Einwohner per $\mathrm{km}^{2}$ aufweisen. Aber auch die fruchtbaren Gebiete des Garonnebeckens und das südliche Rhonegebiet sind relativ bevölkerungsarm. Von den 24. Departementen, deren Volksdichtewerte das Mittel von 76,1 übersteigen, gibt es nur 13, deren Volksdichte größer als 100 ist, und davon nur 6, die mehr als 150 Einwohner per $\mathrm{km}^{2}$ aufweisen, also nur $7 \%$, während in der Schweiz 9 Kantone oder $36 \%$ die gleiche Dichte erreichen.

Nach Untersuchungen von A. Demangeon und M. Matruchot ${ }^{3}$, die sich auf die fast 3000 Kantone Frankreichs erstrecken, ergibt sich, daß in der Zeit von 1881 bis 1921 von 2957 Kantonen (ohne Elsaß-Lothringen) nur 617 an Einwohnern zugenommen, dagegen 2344 oder $80 \%$ abgenommen haben, und zwar von diesen $18 \mathrm{um}$ mehr als $50 \%$; die Mehrzahl derselben liegt allerdings in der Kriegszone. In 18 Departementen haben alle Kantone Bevölkerungsverluste erlitten, nämlich in den Departementen der Alpen, der Pyrenäen und des Zentralmassivs, ferner in den östlichen und zentralen Kalkplateaus und sogar in dem flachen, milden Becken von Aquitanien.

Ähnliche Beträge eines auffallend starken Bevölkerungsrückganges lassen sich aber auch für ganze Departemente feststellen, wenn man ihre Bevölkerungsbewegungen in der Zeitspanne von 1891 bis 1931, eventuell bis 1946 untersucht. So gehören von den 66 Departementen, die seit 55 und mehr Jahren fast beständig abgenommen haben, die folgenden $24 \mathrm{zu}$ denen mit größtem Bevölkerungsrückgang, was in den beigefügten Prozentzahlen deutlich zum Ausdruck kommt: Somme 21\%, Aveyron $22 \%$, Dordogne $23 \%$, Eure und Nièvre je $24 \%$, Cantal $25 \%$, Ardennes $26 \%$, Manche $27 \%$, Haute-Marne und Yonne je $30 \%$, Jura und Mayenne je $31 \%$, Tarn et Garonne 32\%, Creuse $33 \%$, Ardèche und Hautes-Alpes je $35 \%$, Lozère und Orne je $37 \%$, Gers $38 \%$, Haute-Saône $41 \%$, Meuse $42 \%$, Ariège und Basses-Alpes je $45 \%$ und Lot $47 \%$.

Im Gegensatz zu diesen ausgesprochen agrarischen und meist von großen Verkehrslinien gemiedenen ländlichen Departementen weisen in der gleichen Zeit die industriereichen oder verkehrsbegünstigten mit großen Städten ganz erhebliche $\mathrm{Zu}$ nahmen ihrer Bevölkerung auf. Von den 22 Departementen mit Zunahme seien hier diejenigen genannt, deren Bevölkerung um 10 bis $54 \%$ zugenommen hat, nämlich: Hérault, Seine-et-Marne, Nord, Rhône, Meurthe-et-Moselle, Pas-de-Calais, Seine-etOise, Seine, Bouches-du-Rhône und Alpes-Maritimes. Der Leser erkennt ohne weiteres $\mathrm{da} B$ es sich u. a. um Gebiete handelt, in denen sich die größten Städte Frankreichs befinden. Auch die Städte Bordeaux, Toulouse, Cherbourg, Perpignan liegen in den durch etwas schwächere Zunahme charakterisierten Departementen. Die von 1921 bis 1931 in Zunahme begriffenen Regionen Frankreichs sind von A. CHOLLEY ${ }^{4}$ auf einer

3 "Les variations de la population en France de 1881 à 1921." Annales de Géographie, 35, 1926, p. 499-510.

4 "La population de la France en 1931." Annales de Géographie, 41, 1932, p. 638-640. 
Karte wiedergegeben worden. Auffallenderweise ist gerade in mehreren dieser dichtbevölkerten Städte-Departemente von 1931 bis 1946 ein nicht unbedeutender Rückgang von 5 bis $15 \%$ eingetreten, so in den Departementen: Alpes-Maritimes, Bouchesdu-Rhône, Nord, Pas-de-Calais, Seine und Seine-Inférieure, Moselle und HauteVienne $^{5}$. Es handelt sich hierbei vorherrschend um Gebiete, die von Ausländern stark bevölkert waren, welch letztere aber infolge des zweiten Weltkrieges größtenteils gezwungen wurden, das Land zu verlassen.

Diese Tatsache widerlegt jedoch nicht die seit Jahrzehnten beobachtete allgemeine Erscheinung, daß das Land sich beständig mehr entvölkert, während die großen Städte zunehmen. Zu dieser "Flucht nach der Stadt» gesellt sich das seit vielen Jahrzehnten übliche System der absichtlichen Kinderbeschränkung der französischen Familien, ein System, das sich in ganz erschreckend nachteiliger Weise auf die Landbevölkerung auswirkt.

Aber während nach Sconess Handbuch ${ }^{6}$ um 1891 die Zahl der Kinder in der französischen Familie durchschnittlich noch nicht 3 (genau 2,91) betrug, wurde sie für die Jahre 1926 und 1936 auf nur 1,8 berechnet. Es werden dabei 10 Departemente aufgeführt mit einer mittleren Kinderzahl von 2,2 bis 2,7, denen 10 andere mit einer Kinderzahl von nur 1,2 bis 1,6 gegenüberstehen ${ }^{7}$. Der Vergleich der beiden Jahre 1926 und 1936 zeigt, daß die mittlere Kinderzahl seit 1926 eher zurückgegangen ist; aber auch der Prozentsatz der kinderlosen Familien hat sich in dieser Zeit etwas verringert, nämlich von 23,3 auf 22,8\%. Ferner hat der Prozentsatz der kinderreichen Familien ( 7 und mehr Kinder) von 1926 auf 1936 leicht abgenommen, von 2,3 auf 2,1\% im Mittel.

Den Folgen des Ein- und Zweikindersystems und weiteren Ursachen der allgemeinen Entvölkerung ist L. NUDEAU mit ganz besonderem Eifer und Sachkenntnis nachgegangen, und es ist nur zu bedauern, daß seinen anregenden Ausführungen nicht mehr Beachtung geschenkt wurde ${ }^{8}$. Aus ihnen geht hervor, daß das Problem ein sehr komplexes ist und daß eine Reihe von Tatsachen zur Erklärung der beunruhigenden Erscheinungen beigezogen werden muß. So dürfen ohne Zweifel die vielen Kriege, die Frankreich sowohl im 19. wie im 20. Jahrhundert zu bestehen hatte und die vom Land ungeheure Blutopfer forderten, in sehr bedeutendem Umfange an der Bevölkerungsabnahme schuld sein. Dazu kommen in vielen Gebieten, Dörfern und Städten, infolge unhygienischer Wohnungen ansteckende Krankheiten, namentlich Tuberkulose, an denen viele Leute vorzeitig zugrunde gehen, ebenso an Alkoholismus. Ergreifend sind beispielsweise Schilderungen Nudeaus von den Arbeiterwohnungen, den sogenannten "taudis» in Alt-Rouen, wo namentlich unter den kinderreichen Arbeiterfamilien eine große Kindersterblichkeit herrscht («Illustration» vom 8. März 1930). Nicht weniger eindrucksvoll sind Nudeaus Beschreibungen von traurigen Wirtschaftsund Wohnverhältnissen auf dem Lande, wo man fast in jedem Dorf auf eine ganze Anzahl verlassener Höfe und zusammengefallener Häuser stößt. Die seit der französischen Revolution eingeführte Aufteilung größerer Landgüter hat viele ehemalige Pächter zu Kleinbauern gemacht, die bei schwerer Arbeit und geringen Einkünften unter primitiven Lebensverhältnissen existieren müssen; diesen vermögen ihre Kinder keinen Geschmack abzugewinnen, und sie ziehen der eigenen Scholle eine Anstellung in der Stadt vor, die ihnen ein bequemeres und angenehmeres Leben verspricht.

Nach M. AllanN ${ }^{9}$ sind $80 \%$ der Bauern Frankreichs Landbesitzer; wenn es auch in etwa einem Dutzend Departementen noch über 100 Güter von je 200 ha und mehr

5 Annales de Géographie, 56, 1947.

- A. Scoвel: "Geographisches Handbuch zu Andrees Handatlas." 2. Auflage. Bielefeld 1895.

7 G. Mauco: "La statistique des familles en 1936." Annales de Géographie, 55, 1946, p. 238-240.

${ }^{8}$ Enquête sừ la population de la France. "L'Illustration», 1929 et 1930, Paris.

- "Géographie Universelle», tome 2. Paris 1923. 
gebe, wobei es sich meist um weniger abträgliches Land handle, so betrage doch die mittlere Größe der Bauerngüter nur 8;65 ha (rund 26 Jucharten), und über 2,5 Millionen Bauern besäßen kaum 1 ha eigenes Land. Ferner sei das Land der mittelgroßen Güter infolge der Erbverhältnisse in der Regel sehr zerstückelt, in schmale Riemen aufgelöst und daher ihre Bebauung zeitraubend.

In den Jahren 1870 bis 1880 machte die Landwirtschaft Frankreichs eine ernste Krise durch, indem zufolge des entwickelten Land- und Seeverkehrs der Markt mit ausländischen landwirtschaftlichen Produkten aller Art überschwemmt wurde. Dadurch wurden die Preise der einheimischen Produkte so stark gedrückt, daß der Bauer bei den hohen Steuern kaum mehr bestehen konnte.

Die ökonomischen Verhältnisse der französischen Landwirte sind auch seither nicht viel besser geworden. Der Abgang von Hilfskräften infolge der beiden Weltkriege und des Zuges nach der Stadt, und die durch niedrige Zölle nicht genügend abgehaltene Einfuhr ausländischer Produkte sowie der blühende Zwischenhandel verhindern eine wirkliche Prosperität der französischen Landwirtschaft. So liegen sehr große Flächen fruchtbarsten Acker- und Wiesenlandes in Frankreich unbebaut und werden vom Unkraut überwuchert, während die Regierung große Anstrengungen macht, um aus Steppen französischer Kolonien karges Kulturland zu schaffen!

Viele junge Franzosen verlassen das Land, um in den Kolonien oder anderswo ihr Glück zu suchen; an ihre Stelle treten zahlreiche Fremde. In den Jahren 1922 bis 1932 betrug die mittlere jährliche überseeische Auswanderung 62000 Franzosen, während in der gleichen Zeit jährlich 159000 Ausländer in Frankreich einwanderten.

Ohne Zweifel liefert die offizielle Statistik nicht ein genaues Bild der Fremdbevölkerung in Frankreich; denn die im Lande geborenen Kinder der Ausländer werden ohne weiteres naturalisiert, gelten also als Franzosen. Im Jahre 1870 zählte man 740700 oder $2 \%$ Fremde, im Jahre 1891 war die Million überstiegen mit 2,8\%, und 1931 wurden 2,89 Millionen oder $6 \%$ der Bevölkerung als Ausländer gezählt. Unter diesen Ausländern stehen Italiener, Spanier, Belgier und Polen weit obenan. Während die beiden ersteren Volksgruppen hauptsächlich im Süden tätig sind, beschäftigt man Belgier und Polen in erster Linie in den Minengebieten des Nordostens. Nach der Statistik machen die Ausländer $10 \%$ der Bevölkerung im Norden und in Paris aus, $18 \%$ im Moselgebiet, 21,8\% im Departement Bouches-du-Rhône und $29 \%$ in den AlpesMaritimes. Daß heute schon vielfach Grund und Boden in den Besitz von Ausländern übergegangen ist, ist eine andere bemerkenswerte Tatsache, die sich zwangsläufig aus den beschriebenen Verhältnissen ergibt. Als weitere Folge dieser Zunahme von Ausländern muß im Laufe der Zeit eine Veränderung und Umbildung des französischen Volkstums eintreten.

\section{PROBLEMES DE LA POPULATION FRANÇAISE}

On a constaté que, depuis un siècle en France, dans un grand nombre de départements, surtout dans les régions rurales, la population a constamment diminué, tandis que celle des grandes villes a fortement augmenté. Aussi, le faible accroissement de la population totale est-il dû exclusivement à l'immigration étrangère: le chiffre de 740700 étrangers (soit $2 \%$ ) en 1870 est monté à 2,87 millions en 1931 (soit 6\%).

\section{PROBLEMI DEMOGRAFICI DELLA FRANCIA}

Negli ultimi cento anni in molti dipartimenti francesi, specie in quelli agrari, si è verificata una diminuzione continua della popolazione, mentre il numero degli abitanti di città aumentava. L'accrescimento debole della popolazione totale è dovuta principalmente all'immigrazione.

Con 740000 persone nel 1870 gli stranieri costituivano il $2 \%$ della popolazione totale, mentre nel 1931 con 2,87 milioni questi ne costituivano il $6 \%$. 